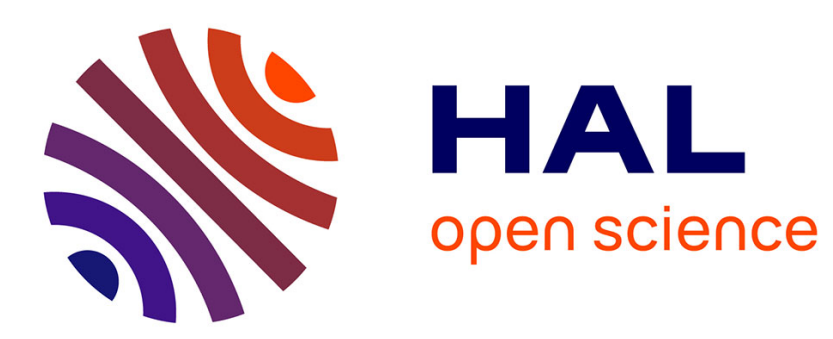

\title{
Law of spreading of the crest of a breaking wave
}

Yves Pomeau, Thimotée Jamin, Michael Le Bars, Patrice Le Gal, Basile

Audoly

\section{To cite this version:}

Yves Pomeau, Thimotée Jamin, Michael Le Bars, Patrice Le Gal, Basile Audoly. Law of spreading of the crest of a breaking wave. Proceedings of the Royal Society of London Series A Mathematical and Physical Sciences, 2008, 464, pp.1851. 10.1098/rspa.2008.0024 . hal-00426015

\section{HAL Id: hal-00426015 https://hal.science/hal-00426015}

Submitted on 23 Oct 2009

HAL is a multi-disciplinary open access archive for the deposit and dissemination of scientific research documents, whether they are published or not. The documents may come from teaching and research institutions in France or abroad, or from public or private research centers.
L'archive ouverte pluridisciplinaire HAL, est destinée au dépôt et à la diffusion de documents scientifiques de niveau recherche, publiés ou non, émanant des établissements d'enseignement et de recherche français ou étrangers, des laboratoires publics ou privés. 


\title{
Law of spreading of the crest of a breaking wave
}

\author{
Y. Pomeau ${ }^{\mathrm{a}, *}$, T. Jamin $^{\mathrm{b}}$, M. Le Bars ${ }^{\mathrm{b}}$, \\ P. Le Gal ${ }^{\text {b }}$ and B. Audoly ${ }^{\mathrm{c}}$ \\ ${ }^{\mathrm{a}}$ Laboratoire de Physique Statistique, École Normale Supérieure, 24 rue Lhomond, \\ 75231 Paris Cédex 05, France. \\ ${ }^{\mathrm{b}}$ Institut de Recherche sur les Phénomènes Hors Équilibre, UMR 6594, CNRS E \\ Aix-Marseille Université, 49 rue F. Joliot-Curie, BP146, 13384 Marseille Cédex \\ 13, France. \\ ${ }^{\mathrm{c}}$ Laboratoire de Modélisation en Mécanique, UMR 7607, Université Pierre et \\ Marie Curie, 4 Place Jussieu, case 162, 75252 Paris Cédex 05, France.
}

\begin{abstract}
In a wide range of conditions ocean waves break. This can be seen as the manifestation of a singularity in the dynamics of the fluid surface, moving under the effect of the fluid motion underneath. We show that, at the onset of breaking, the wave crest expands in the span-wise direction as the square root of time. This is first derived from a theoretical analysis and then compared with experimental findings. The agreement is excellent.
\end{abstract}

\section{Introduction}

Wave-breaking in shallow water may be seen as the expression in the real world of the occurrence of a singularity in the solution of the equations of motion for the free surface. Somewhat related ideas have been presented by Whitham (1974), without however making predictions for the widening of the rolling crest. The wave-breaking is a singularity because this set of equations is derived under the assumption that the surface is close to horizontal, and because its solution after a finite time has a vertical tangent plane. This problem is studied first in a model, assumed to be generic for this type of singularity:

\footnotetext{
* corresponding author

Email address: pomeau@lps.ens.fr (Y. Pomeau).
} 
the so-called Burgers equation. This yields generic scaling laws for various quantities near the singular time. Usually the analysis of this model is limited to dependence with respect to one space coordinate and to time. This is not sufficient for the breaking of real waves involving deformations of a $2 \mathrm{D}$ surface and needing therefore two space coordinates. Just after breaking this surface includes a closed non planar curve where its tangent plane is vertical, this curve growing from a point at the onset of singularity. In a hypothetical $2 \mathrm{D}$ world the study of the $1 \mathrm{D}$ Burgers equation would apply to the typical situation for wave-breaking and the curve just mentioned would reduce itself to a pair of points. In our 3D world, the wave breaking occurs generically at a point on a surface and spreads later on in the span-wise direction. This paper gives the time dependence of this spreading. Real waves are described by highly nonlinear equations without hope of any explicit general solution. Therefore we rely on an analysis of the breakdown of smooth solutions of model equations with two space coordinates. In this model the width of the singular region increases generically like the square root of time difference between the actual time and the instant where the solution becomes singular first. Then we show theoretically that the same breaking process occurs in 'real' water wave, in the approximation of long wave, relatively small amplitude and negligeable dispersion. In the last part we present experimental observations of the spreading of the crest of breaking-wave in a laboratory set-up allowing accurate measurements in shallow water, this showing a remarkable agreement with the theoretically predicted exponent.

\section{From 1D to 2D wave-breaking: a model}

Below we first recall standard results on the so-called one dimensional (1D) inviscid Burgers equation and extend them to two space dimensions (2D). The main result of the $2 \mathrm{D}$ analysis is that the width of the singular region grows like the square root of time, after the first singularity.

In 1808 Poisson explained how to solve the Cauchy problem for the equation

$$
\frac{\partial u}{\partial t}+u \frac{\partial u}{\partial x}=0
$$

Thanks to his solution it is possible to show the occurrence of singularities after a finite time for a wide class of smooth initial data. The equation (1) has the implicit solution

$$
u(x, t)=u_{0}(x-u t)
$$

where $u_{0}(x)$ is the initial condition, taken at $t=0$. Note that (i) in the 
following, we change the origin of time so that the first singularity in the solution of Burgers equation takes place at $t=0$ (the initial condition is then somewhere at $t<0$ ) and that (ii) we do not specify what this initial condition is (we only need it to be bell-shaped and smooth).

Another way of writing this solution is to consider the argument of $u_{0}$, on the right-hand side of equation (2), as a function of $u(x, t)$ :

$$
f(u(x, t))=x-u t
$$

where $f($.$) is the inverse function of u_{0}($.$) . It is a classical result that for any$ bell-shaped and smooth initial data $u_{0}$, the first singularity of $u(x, t)$ appears at the inflection point of the function $u_{0}$ on the right of the maximum when $u_{0}$ is positive (see figure 1 ).

Indeed, the singularity happens when the solution $u(x, t)$, as given by equation (3), becomes multivalued, that is whenever $\frac{\partial x}{\partial u}=0$, the derivative being taken

(a)

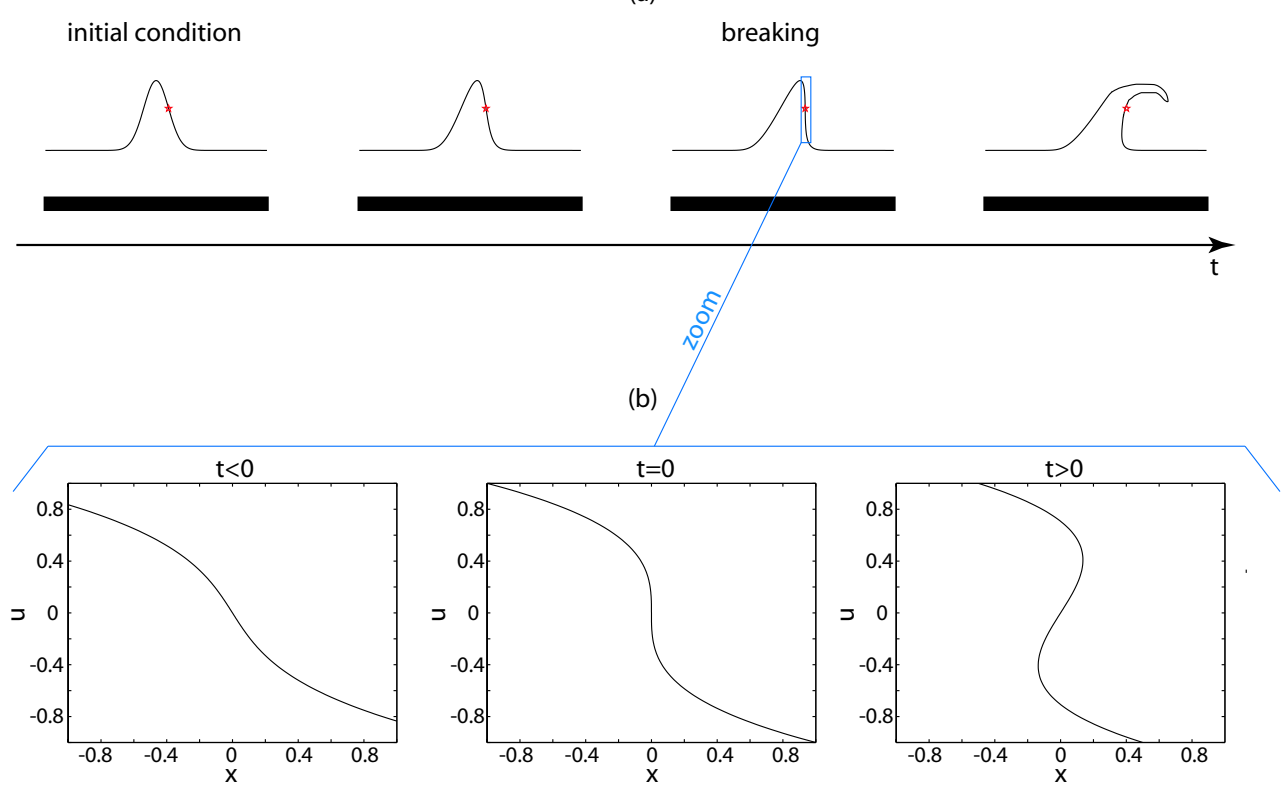

Fig. 1. (a) Sketch of the typical time evolution of a 1D breaking wave, starting for instance from a gaussian profile. The star indicates the inflection point where the singularity first appears. (b) Resolution at three different time of the universal equation for the 'wave-breaking' (5), valid at first order for any bell-shaped and smooth initial condition, considering that the first singularity takes place at $t=0$ and $x=0$ with $u=0$ (after changing the origins of time and space and after Galilean transform). 
at constant $t$. This yields

$$
t+\frac{\mathrm{d} f}{\mathrm{~d} u}=0
$$

For a given $f(u)$, the smallest time when equation (4) has a solution is when this solution is stationary with respect to $t$, that is when $\mathrm{d} t=0, t$ and $u$ being related by (4). This yields $0=\mathrm{d} t=-\mathrm{d} u \frac{\mathrm{d}^{2} f}{\mathrm{~d} u^{2}}$, or simply $\frac{\mathrm{d}^{2} f}{\mathrm{~d} u^{2}}=0$. Let us now look at the Taylor expansion of $f(u)$ around this point. Constant and linear terms can be eliminated by changing the origins of time and space, and by Galilean transform (i.e. the first singularity takes place at $t=0$ and $x=0$ with $u=0)$. Therefore, the Taylor expansion of $f(u)$ reads $f(u)=$ $-u^{3}+a u^{4}+\ldots$, the coefficient of $u^{3}$ being negative to have a singularity appearing in forward times. Once put into equation (3) this gives at lower order the universal equation for the 'wave-breaking', valid for any bell-shaped and smooth initial condition at $t_{0}<0$ :

$$
0=u^{3}+x-u t
$$

For short times (i.e. around the breaking time $t=0$ ), $u$ scales like $t^{1 / 2}$ and $x$ like $t^{3 / 2}$, this making of the same order, $t^{3 / 2}$, all terms on the right-hand side of equation (5).

Note that the result $u(x)$ of $(5)$ is single-valued for $t$ negative and triple-valued for $t$ positive (see figure 1). If a small dissipative term is added to the equation (1) to make it resemble the Navier-Stokes equation, one obtains the so-called Burgers equation:

$$
\frac{\partial u}{\partial t}+u \frac{\partial u}{\partial x}=\epsilon \frac{\partial^{2} u}{\partial x^{2}}
$$

where $\epsilon$ is a small and positive coefficient. The addition of this dissipative term is a way of regularizing the solutions of (1), the multi-valued results being replaced by a jump around $x=0$, that represents a shock for this model.

The novelty of our study comes from the extension of this standard approach to the problem of formation of shocks in solutions of nonlinear hyperbolic equations with more than one space variable. Consider for instance the case of two space variables, $x$ and $y$, and let $u(x, y, t)$ and $v(x, y, t)$ be the two Cartesian components of the velocity field. The generalization of the nonlinear equation (1) reads

$$
\frac{\partial u}{\partial t}+u \frac{\partial u}{\partial x}+v \frac{\partial u}{\partial y}=0
$$


and

$$
\frac{\partial v}{\partial t}+u \frac{\partial v}{\partial x}+v \frac{\partial v}{\partial y}=0
$$

This represents the 'fluid mechanics' equations for a gas without pressure, the origin of which can be traced back to the Principia, Scholium at the end of Proposition IV, problem II, Book 2, where Newton derives the quadratic law of drag for bodies moving in a fluid 'void of tenacity' in the words of the English translation by Motte (the newer translation by Cohen and Whitman translates the original latin into 'lacking in rigidity'). This set of equations has been used by Zel'dovich (1970) to explain the formation of large scale structures in the Universe, assuming a potential flow. We now suggest that it provides a model for the occurrence of singularities for time-dependent functions of two space coordinates $(x, y)$.

Similarly to the 1D case, equations (7) and (8) admit a formal solution

$$
x-t u(x, y, t)=F(u, v),
$$

and

$$
y-t v(x, y, t)=G(u, v)
$$

where $F(u, v)$ and $G(u, v)$ are defined by the initial conditions. A crucial remark for the following is that the left-hand sides of the equations (9) and (10) are invariant under general transformations of the linear group. Let $\mathcal{M}$ be the general two by two matrix of this group, with non-zero determinant. It is obvious that $(\tilde{u}, \tilde{v})=\mathcal{M}(u, v)$ and $(\tilde{x}, \tilde{y})=\mathcal{M}(x, y)$ are solutions of equations of the same form as the left-hand side of equations (9) and (10). This holds true with the same matrix $\mathcal{M}$ acting on $(u, v)$ and $(x, y)$. Of course the same general linear map will change in general the functions $F(u, v)$ and $G(u, v)$ in a rather complicated way. But, because the map is linear it will not change the degree of a polynomial in $u$ and $v$. Thanks to this remark we shall be able to show, without calculation, that singularities yielding discontinuities of derivatives with respect to $x$ may spread in any direction, the analysis being made by assuming first that the singularity spreads normal to $x$.

For smooth initial data, $F$ and $G$ are such that the initial mapping from $(u, v)$ to $(x, y)$ is one to one. The solutions of equations (7) and (8) become singular whenever the time dependent Jacobian $J(t)$ of the mapping from $(u, v)$ to $(x, y)$ becomes singular at a certain time $t$. This Jacobian reads:

$$
J(t)=\left(\frac{\partial G}{\partial v}+t\right)\left(\frac{\partial F}{\partial u}+t\right)-\frac{\partial G}{\partial u} \frac{\partial F}{\partial v} .
$$


Let us assume that the singularity occurs first at $t=0$ and at $x=y=0$ with $u=v=0$ too. As in the $1 \mathrm{D}$ case, this last restriction is not as important as one could believe first: the original equations are Galilean invariant, so that one can always bring to $u=v=0$ the velocity field at some point of time and space. The condition equivalent to the cancelation of the second derivative of $f(u)$ at $u=0$ here is the property that, near $t=u=v=0$, the first non-vanishing terms in the Taylor expansion of the Jacobian $J(t)$ read

$$
J(t)=a t+b u^{2}+c v^{2}+2 d u v+\ldots
$$

where $a, b, c$ and $d$ are constants derived from initial conditions. To give the quadratic term $\left(b u^{2}+c v^{2}+2 d u v\right)$ in the Jacobian, $F(u, v)$ and $G(u, v)$ have to be expanded in Taylor series to third order, which depends on two sets of ten coefficients. But the algebra is greatly simplified by limiting oneself to the leading order terms near the singularity. Indeed, at $t=0$ the Jacobian matrix has a zero eigenvalue that can be associated to an eigenvector pointing in the $x$ direction. Therefore the coefficients of the Taylor expansion of $F(u, v)$ and $G(u, v)$ are such that $\frac{\partial F}{\partial u}=0, \frac{\partial F}{\partial v}=0$ and $\frac{\partial G}{\partial u}=0$ at $u=v=0$. This yields that, near the singularity, the leading order term in $F(u, v)$ and $G(u, v)$ should be

$$
F(u, v)=b^{\prime} v^{2}+d^{\prime} u^{3}+g^{\prime} u v^{2}+f^{\prime} u^{2} v \quad \text { and } \quad G(u, v)=c^{\prime} v
$$

where the various coefficients can be easily related to $a, b, c, d$ through equation (12). Then, at leading order, equations (9) and (10) become

$$
\begin{aligned}
& x-u t=b^{\prime} v^{2}+d^{\prime} u^{3}+g^{\prime} u v^{2}+f^{\prime} u^{2} v \\
& y=c^{\prime} v
\end{aligned}
$$

the term $t v$ in (10) being negligible compared to $c^{\prime} v$. Further simplifications of equation (14) are now possible. First, one can notice that the term $b^{\prime} v^{2}=$ $\left(b^{\prime} / c^{\prime 2}\right) y^{2}$ changes the support of the singularity from a straight line to a bent line without playing any other significant role: near the singularity one may always approximate the line of singularity by a straight line. Second, one can also neglect the term $f^{\prime} u^{2} v=\left(f^{\prime} / c^{\prime}\right) u^{2} y$, since it can be absorbed into a change of $u$ into $u+e y, e$ being a constant. After rescaling, equation (14) finally leads to a universal equation for the 'wave-breaking' in 2D, valid for any bell-shaped and smooth intial conditions:

$$
0=u^{3}+x-u\left(t-y^{2}\right) .
$$

Note that all terms in equation (16) are of order $|t|^{3 / 2}$ near the singularity, with $y \sim|t|^{1 / 2}, u \sim|t|^{1 / 2}$ and $x \sim|t|^{3 / 2}$. This shows our main point, namely that 
the crest of the breaking wave (if this theory applies to the real phenomenon of wave-breaking) spreads like $\sqrt{t}$ after the inception of the singularity, something that we will now verify in a real 'fluid mechanics' configuration.

Let us end this section with three remarks.

i) Note that $x$ and $y$ do not have to be coordinates along orthogonal axis, because the Jacobian matrix is reduced to its diagonal form by a general change of coordinates that is not necessarily a rotation, the Jacobian being not real symmetric in general.

ii) Supposing that $u(x, y, t)$ behaves in the same way as the vertical position $z(x, y, t)=u(x, y, t)$ on the surface of a breaking wave (which is the case in shallow water, see next section), one finds the loci of points of vertical slope on the surface (the contour of the surface) by deriving from equation (16) the coordinates of the points where $\mathrm{d} z=0$. The projection on the horizontal plane of this curve has Cartesian equation

$$
27 x^{2}=4\left(t-y^{2}\right)^{3}
$$

although the $z$ coordinate is such that $z^{2}=\frac{t-y^{2}}{3}$, the determination $z=\sqrt{\frac{t-y^{2}}{3}}$ being taken when $x$ is positive and $z=-\sqrt{\frac{t-y^{2}}{3}}$ with $x$ negative, all this because $x=\frac{2 u}{3}\left(t-y^{2}\right)$ and $t>y^{2}$.

iii) In higher space dimensions, practically in $3 \mathrm{D}$, the reasoning above still works, at least in its general lines. The first and most obvious situation is the onset of formation of a shock wave in a compressible gas (this shock wave could be generated by pushing impulsively a parabolic metal sheets in a gas, leading to the formation of a shock wave near the focus), supposing none exists before the critical time $t=0$. A simple extension of the arguments presented shows that the field $u(x, y, z, t)$, solution of the pressure-less Bernoulli equations becomes singular at one point in the $3 \mathrm{D}$ geometrical space and at a definite time, taken as zero. Locally this field is given by a root of the equation:

$$
0=u^{3}+x-u\left(t-y^{2}-z^{2}\right) .
$$

In this equation $y$ and $z$ are coordinates in a system of oblique axis depending on the initial conditions. In the physical 3D space with the regularization by diffusion the singularity is supported by an ellipse expanding homothetically with a size increasing as $\sqrt{t}$. This would be the case at the onset of formation of a generic shock wave in a compressible gas. But there is more than that in the above analysis. One can also study the case where the coefficient of $u$ has signs different from the ones in equation (17). Consider what happens if this coefficient is $\left(t-y^{2}+z^{2}\right)$ instead of $\left(t-y^{2}-z^{2}\right)$. For any value of $t$ the equation for $u$, namely $0=u^{3}+x-u\left(t-y^{2}+z^{2}\right)$ has three solutions, at least in certain 
domains of the $(x, y, z)$ space, domains that collapse to a surface under the Maxwell construction, a surface making the shock-wave. It means that there is a shock-wave supported by a surface before and after $t=0$. The edge of the shock-wave surface is where and when the coefficient of $u$ is zero, that is along the hyperbola $t=y^{2}-z^{2}$. At $t=0$ this hyperbola degenerates into its two straight asymptotes, $y= \pm z$. On a larger scale there are one or two shock surfaces that may either merge to a single point or break in two pieces, depending on what side of the hyperbolae lies the shock. The local hyperbolae are the edges of the shock surface, the jump across the shock tending to zero as one moves along the shock surface toward this edge. A hard to read paper by Arnold et al. (1990) deals with a related topic, the bifurcations of a shock surface.

\section{Wave breaking in shallow water}

The analysis presented before does not apply directly to the case of 'real' water waves, because they are not described mathematically by the equations for a fluid void of tenacity, or pressure-less. However it can be shown, as done below, that, in a well-defined limit, this theory is relevant for the breaking of waves in shallow water. This is done by estimating the typical quantities describing the wave-breaking in a way inspired by the pressure-less case, and by showing that, in a reference frame moving at the speed of linear waves, one recovers the equations of the pressure-less fluid near the singularity.

The dynamics of long waves in a layer of inviscid fluid of depth much smaller than the typical range of variation of the various quantities involved (see section 4 for a discussion of the order of magnitudes involved) can be described by the following set of equations for the basic quantities corresponding to the fluid depth $h(x, y, t)$ and to the two Cartesian components of the horizontal fluid velocity, $u(x, y, t)$ and $v(x, y, t)$ : the condition of mass conservation reads

$$
\frac{\partial h}{\partial t}+\frac{\partial(u h)}{\partial x}+\frac{\partial(v h)}{\partial y}=0
$$

the condition of momentum conservation in the $x$-direction reads

$$
\frac{\partial u}{\partial t}+u \frac{\partial u}{\partial x}+v \frac{\partial u}{\partial y}+g \frac{\partial h}{\partial x}=0
$$

and in the $y$-direction

$$
\frac{\partial v}{\partial t}+u \frac{\partial v}{\partial x}+v \frac{\partial v}{\partial y}+g \frac{\partial h}{\partial y}=0
$$


In equations (19) and (20), $g$ is the positive acceleration of gravity. This set of equations admits non-trivial simple wave solutions whenever no function depends on $y$ (for instance). Non-trivial solutions including both a dependence on $x, y$ and $t$ do not seem to exist. Nevertheless it is possible to analyze the occurrence of singularities by solving this set in a convenient limit.

Let us do it first for solutions depending on $x$ only. We assume that $h=$ $h_{0}+\tilde{h}(x, t)$ where $h_{0}$ is the constant (and positive) depth, much larger than the small perturbation $\tilde{h}(x, t)$. This allows to neglects terms of order $\tilde{h}(x, t) u$ with respect to $h_{0} u(x, t)$. The new set of equations reads:

$$
\frac{\partial \tilde{h}}{\partial t}+u \frac{\partial \tilde{h}}{\partial x}+h_{0} \frac{\partial u}{\partial x}=0
$$

and:

$$
\frac{\partial u}{\partial t}+u \frac{\partial u}{\partial x}+g \frac{\partial \tilde{h}}{\partial x}=0
$$

Notice that, although $\tilde{h}$ has been neglected with respect to $h_{0}$ in front of $\frac{\partial u}{\partial x}$, $u \frac{\partial \tilde{h}}{\partial x}$ has been kept, because it is a priori not smaller than any other term written explicitly in equation (21) (in particular, at the singularity, we expect the $x$ derivative to go to infinity).

Take now $h_{0}$ as unit for $\tilde{h}$, introduce $C_{B}$ such that $C_{B}^{2}=g h_{0}$ (the subscript $B$ is to recall that this velocity was derived first by Bernoulli), take $C_{B}$ as unit for $u$ and chose new units for $t$ and $x$ such that their ratio is one when their ratio in physical units is $C_{B}$, the equations for $\tilde{h}$ and $u$ become:

$$
\frac{\partial \tilde{h}}{\partial t}+u \frac{\partial \tilde{h}}{\partial x}+\frac{\partial u}{\partial x}=0
$$

and:

$$
\frac{\partial u}{\partial t}+u \frac{\partial u}{\partial x}+\frac{\partial \tilde{h}}{\partial x}=0 .
$$

Those equations become identical if $u=\tilde{h}$. This corresponds to the so-called simple wave. Let $u=\tilde{h}=w$, the two equations (23) and (24) become the single one:

$$
\frac{\partial w}{\partial t}+w \frac{\partial w}{\partial x}+\frac{\partial w}{\partial x}=0
$$


This is brought back to the familiar (so-called) inviscid Burgers equation (1) by the change of unknown function from $w$ to $(w+1)$. It shows that the singularity is the same as the one found before, of course not a new result at all, because of the possibility of solving the full set (18), (19) and (20) by Riemann's method with $v=0$ and no dependence on $y$.

Let us now look at the dimensionless equations for the nonlinear wave propagation in shallow water in $2 \mathrm{D}$, using the same scaling. The mass conservation yields:

$$
\frac{\partial \tilde{h}}{\partial t}+\left(\frac{\partial u}{\partial x}+\frac{\partial v}{\partial y}\right)+u \frac{\partial \tilde{h}}{\partial x}+v \frac{\partial \tilde{h}}{\partial y}=0
$$

The conditions of momentum conservation along $x$ and $y$ read:

$$
\frac{\partial u}{\partial t}+u \frac{\partial u}{\partial x}+v \frac{\partial u}{\partial y}+\frac{\partial \tilde{h}}{\partial x}=0
$$

and

$$
\frac{\partial v}{\partial t}+u \frac{\partial v}{\partial x}+v \frac{\partial v}{\partial y}+\frac{\partial \tilde{h}}{\partial y}=0
$$

Let us assume first that the scaling laws for $y, x, t$ and $u$ are the same as in the generic singularity of the 2D Burgers equation, that is $y \sim|t|^{1 / 2}, x \sim|t|^{3 / 2}$ and $u \sim v \sim|t|^{1 / 2}$. Then in equation (26), $\frac{\partial u}{\partial x} \sim|t|^{-1}$ is dominant compared to $\frac{\partial v}{\partial y} \sim|t|^{0}$. Therefore at leading order equation (26) becomes:

$$
\frac{\partial \tilde{h}}{\partial t}+\frac{\partial u}{\partial x}+u \frac{\partial \tilde{h}}{\partial x}+v \frac{\partial \tilde{h}}{\partial y}=0
$$

Similarly to the $1 \mathrm{D}$ case, we can then look for a simple wave solution, i.e. $\tilde{h}=u$. Taking $w=u+1$ as new unknown function, one finds that (26) and (27) reduce to a single equation for $w$

$$
\frac{\partial w}{\partial t}+w \frac{\partial w}{\partial x}+v \frac{\partial w}{\partial y}=0
$$

Let us now write equation (28) with this new function $w$ :

$$
\frac{\partial v}{\partial t}+w \frac{\partial v}{\partial x}+v \frac{\partial v}{\partial y}-\frac{\partial v}{\partial x}+\frac{\partial w}{\partial y}=0
$$

The first three terms correspond to the 2D Burgers equation, whereas the last two terms correspond to the vertical vorticity. Let us look at their contribution, 
assuming that the scaling from section 2 holds: $\frac{\partial v}{\partial x}$ is then zero, whereas $\frac{\partial w}{\partial y}$ induces a negligeable correction on $v$ of order $t$. This means that starting from an irrotational initial condition (a smooth wave propagating in the $x$ direction with no $y$ dependence), no vorticity generation is to be expected at the first order relevant to our theory. Note however that this does not preclude vorticity generation at higher order at the edge of the breaking zone, as shown for instance by Peregrine (1999) at the edge of bores. This very interesting question is beyond the scope of the present paper, but will be the focus of future theoretical and experimental studies.

Neglecting the vorticity, the system to be solved is the same as the one solved for the pressure less fluid. This proves our point, namely that at leading order the singular solution is for $u($.$) (and so for \tilde{h}$ ) the same as for the pressure less case, up to the addition of the constant speed necessary to get rid of the Galilean change of frame of reference, the fluid velocity $v$ being just proportional to $y$. In particular, the widening of the wave-breaking domain in the $y$ direction scales like the square root of time.

The calculation above suggests that this behaviour is 'universal' for non-linear non-dispersive waves. Other kind of waves are known to break, like the ocean waves on deep water (with, practically, infinite depth) or the gravity waves in the atmosphere. Does their spreading in the $y$ direction follow the same square root law? The answer to this question depends in particular on how precise can be set the instant of time of the breaking process.

\section{Wave-breaking in the 1D case: nonlinearity vs dispersion}

This section is to look more closely at the wave-breaking in 1D. More specifically we make the connection between the solution of the equations (18-20) and the 'real' wave-breaking. The need to do this arises from the simple observation that the long-wave equations are clearly invalid at the time of the break-up and, presumably some time before because the slope $\frac{\partial h}{\partial x}$ then diverges, although the equations are derived under the assumption of a surface with a small slope. This situation is rather common in many problems where the onset of singularity is just a witness that somewhere and sometime another approximation (or another method) has to be used to solve the problem at hand, an approximation different from the one that leads to the singular behaviour.

It is known that dispersion effects occurring at the next order in the shallow water approximation (the small parameter being the fluid depth, the leading order being the equations of the previous section) yield the Korteweg-de Vries $(\mathrm{KdV})$ equation in the comoving frame. The $\mathrm{KdV}$ equation has no finite time 
singularity with smooth initial data and yields instead solitons where the nonlinearity and the dispersion balance each other to avoid singularities. It is worth pointing out that this discussion is a very natural extension of the one made long ago by Stokes to define precisely the range of validity of the linearized Boussinesq theory. It is at least in qualitative agreement with the observation reported in section 5 that waves break or not depending on their amplitude: at small amplitude dispersion dominates and there is no breaking, although at higher amplitudes nonlinear effects dominate and waves break.

The KdV equation in the frame of reference moving with the speed of the waves in the linear approximation, that is $C_{B}=\sqrt{g h_{0}}$, reads:

$$
\frac{\partial \tilde{h}}{\partial t}+A \tilde{h} \frac{\partial \tilde{h}}{\partial x}+B \frac{\partial^{3} \tilde{h}}{\partial x^{3}}=0
$$

where $A=\frac{C_{B}}{h_{0}}$ and $B$ is proportional to $C_{B} h_{0}^{2}$ with a numerical constant irrelevant for this discussion. This equation applies if the non-linear and regularising terms are of the same order of magnitude, which requires

$$
\frac{\tilde{h}}{h_{0}} \sim\left(\frac{h_{0}}{\Lambda}\right)^{2}
$$

where $\Lambda$ is the typical length scale for the variation with respect to $x$ although the amplitude of $\tilde{h}$ is denoted simply as $\tilde{h}$. If on the contrary

$$
\frac{\tilde{h}}{h_{0}} \gg\left(\frac{h_{0}}{\Lambda}\right)^{2},
$$

the nonlinearities dominate the effects of dispersion, and wave-breaking occurs with suitable initial conditions, as demonstrated theoretically in the previous section and as will be seen experimentally in the next section. Note finally that the opposite condition was found by Stokes (1847) for the applicability of the linear approximation for waves propagating in shallow water.

Near the singularity the slope of the surface will diverge so that the long-wave equations are clearly invalid in an inner region. The order of magnitude of the (inner) domain where the shallow water approximation does not apply is found by writing the equation (5) with physical quantities relevant for the wave-breaking problem. The inner region is the neighborhood of $x=t=0$ where the slope $\frac{\mathrm{d} h}{\mathrm{~d} x}$ becomes of order 1 . The equation $f(u)=-u^{3}$ becomes in physical units $f(u)=-\alpha u^{3}$ with $\alpha=\frac{\Lambda}{C_{B}^{3}}\left(\frac{h_{0}}{\tilde{h}}\right)^{3}$. This scaling law is derived by noticing that, near the singularity, $\tilde{h}(x) \sim \tilde{h}\left(\frac{x}{\Lambda}\right)^{1 / 3}$ and that $u \sim \frac{C_{B}}{h_{0}} \tilde{h}$. With the scaling laws derived in Section 2 , one finds that if $\frac{\mathrm{d} h}{\mathrm{~d} x} \sim 1$, then $t \sim t^{*}$ with $t^{*}=\frac{h_{0}}{C_{B}}$. From the scaling $x \sim t^{3 / 2} \alpha^{-1 / 2}$ the width of the inner 
region is $x^{*} \sim \frac{\tilde{h}^{3 / 2}}{\Lambda^{1 / 2}}$. Indeed the scaling laws just derived are only valid until the time of break-up, where another approximation has to be used. In the wavebreaking problem, one expects regularization (something allowing practically to describe what happens beyond the singularity occurring in the shallow water approximation) by taking into account the complete fluid equations instead of their long-wave limit. This regularization changes the local equations near the area where overturning occurs, but it does not change the scaling laws for the extent of this area in time and space. This regularization is purely local, although the large scales remain described by the long wave approximation, making the outer problem. The resulting multi-scale analysis will be presented in a future publication.

\section{Experimental validation: breaking waves in shallow water}

The above theory has been checked against experiments measuring the spanwise widening of the breaking of a wave in shallow water. The experiments have been realized on a $2 \mathrm{~m}$ long and $1.4 \mathrm{~m}$ wide water table. Figure 2 shows this table with its two water tanks and its transparent glass bottom. The level $h_{0}$ of water above the bottom is adjusted to reach the desired values (between 1 and $2 \mathrm{~cm}$ ). The waves are generated by the motion of a rectangular plate (the wave-maker) which is partially immersed in one of the tanks at the end of the table. The controlled motion of this thick PVC plate around an horizontal axis pushes a certain amount of water and so generates a solitary wave that propagates along the table. On a large scale this wave is close to be straight and perpendicular to its direction of motion. The swing motion of the wavemaker is simply induced by its own weight. The generated wave may break or not depending of its height compared to the water depth. The experimental parameters are then adjusted to make the wave break in the center of the working area on the table. To suppress as much as possible reflections of waves on the vertical side-walls, long stripes of plastic foam have been put on the water surface all around the inner walls of the table. To get reproducible waves and consequently a reproducible longitudinal location for the breaking, the starting position of the wave-maker is measured accurately thanks to an optical positioning device and the ending position is fixed by 2 ropes attached on each side of the plate, their length being adjusted to stop the plate motion at the desired value. Underneath the bottom plate made of glass, a translucent screen permits the observation by shadowgraphy of the propagating wave and of its breaking. A light projector is mounted $1.5 \mathrm{~m}$ above the table and form a shadowgraphic image of the deformed water surface on the screen. A mirror inclined at $45^{\circ}$ allows to observe and to record the phenomena occurring inside a square of approximately $32.5 \mathrm{~cm} \times 32.5 \mathrm{~cm}$. A high speed camera with a rate up to $2.8 \mathrm{kHz}$ records the waves and their breaking as they move along the 
table. The optical quality of the system was checked and calibrated by taking the image of a fixed grid.

Experiments have been made with five different depths: $15.5 \mathrm{~mm}, 17.4 \mathrm{~mm}$, $19.7 \mathrm{~mm}$ and $22.5 \mathrm{~mm}$. For each depth, ten runs are performed and recorded. Each experiment corresponds to a different wave height that is measured at the beginning of each run by the use of a sheet of sand-paper held vertically and perpendicular to the wave. This is a simple and easy way to determine quite precisely (precision is about the size of the grains of the sand-paper) the height of the water wave which were equal to respectively $6.3 \mathrm{~mm}, 8.0 \mathrm{~mm}, 10.6 \mathrm{~mm}$ and $9.3 \mathrm{~mm}$ for the previously given water depths. The longitudinal extent of the surface deformation is around $5 \mathrm{~cm}$. These values justify the different approximations of the theoretical study presented in this paper (long wave relatively small amplitude - negligeable dispersion, i.e. $\left(h_{0} / \Lambda\right)^{2}<\tilde{h} / h_{0}<1$, see section 4). Note also that these values fully justify to neglect effects of viscosity and surface tension. Considering the typical viscosity of water (i.e. $10^{-6} \mathrm{~m}^{2} / \mathrm{s}$ ) and the typical duration of one experiment (i.e. $0.3 \mathrm{~s}$ ), the relevant viscous lengthscale is $5.5 \times 10^{-4} \mathrm{~m}$, much smaller than the typical wave size. Similarly, considering the surface tension of water (i.e. $0.073 \mathrm{~N} / \mathrm{m}$ ), the typical capillary length is about $2.7 \times 10^{-3} \mathrm{~m}$, much smaller than the typical wavelength.

Figure 3 shows an example of three consecutive images of a wave travelling

a)

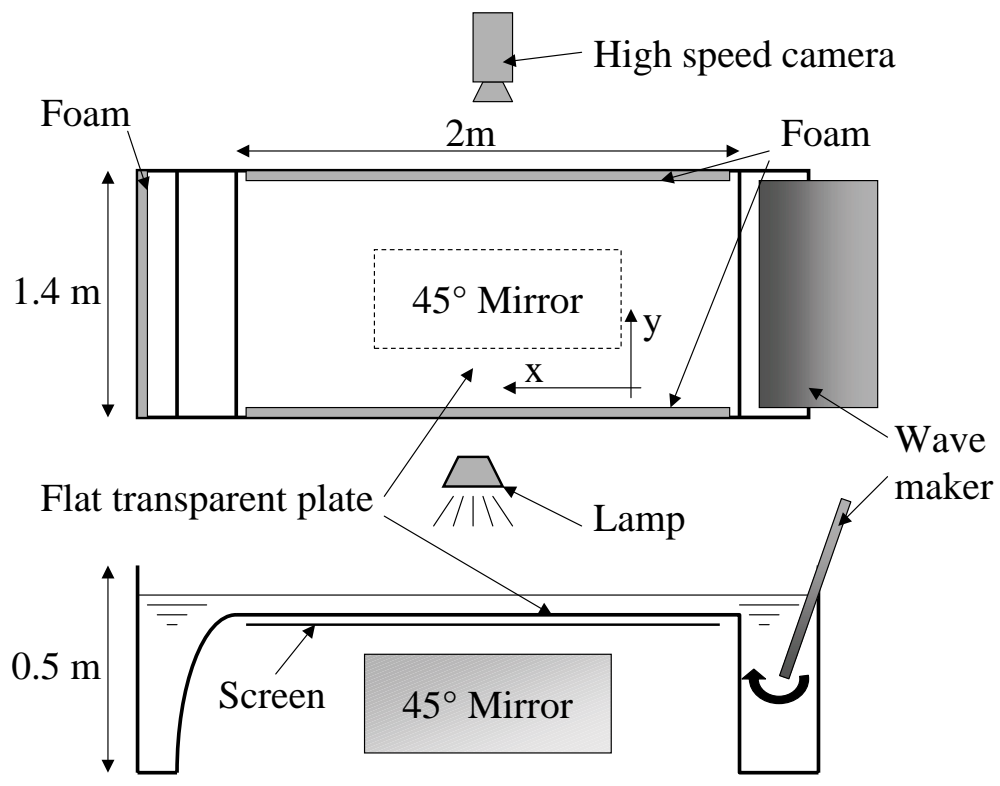

Fig. 2. Experimental set-up, a) top view, b) side view. A solitary wave is generated by the rapid motion of the wave-maker on one side of a water table. The surface deformations, travelling along the longitudinal $x$ direction are visualized by shadowgraphy and recorded by a high speed camera. 
a)

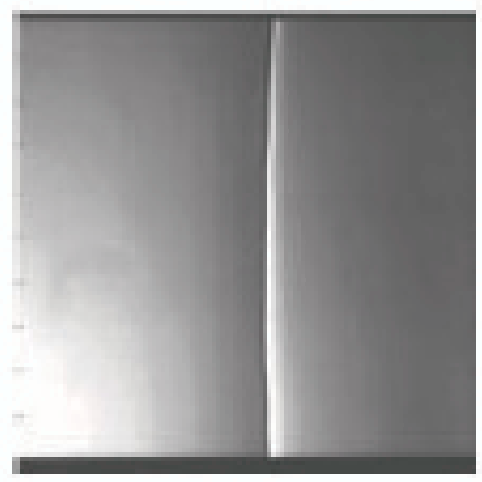

b)

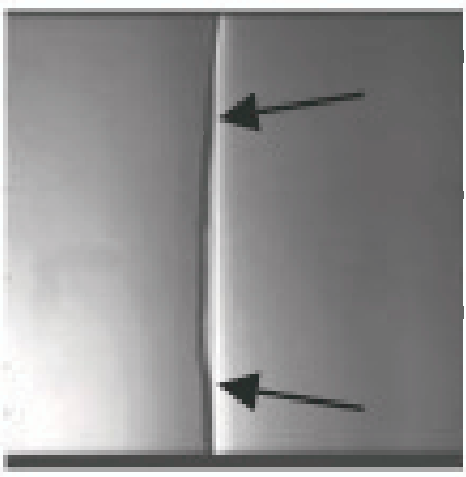

c)

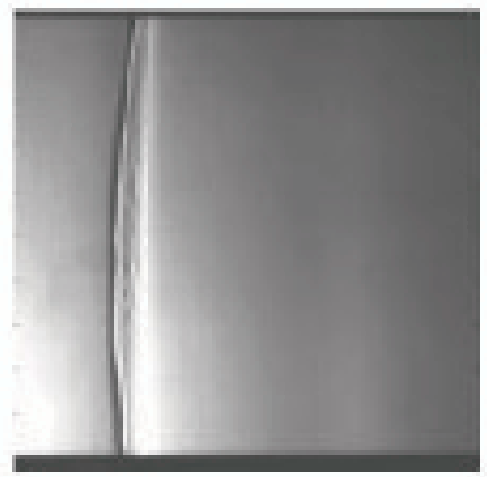

Fig. 3. Shadowgraph of a wave breaking recorded by a high speed camera. a) $t=25 \mathrm{~ms}, \mathrm{~b}) t=100 \mathrm{~ms}, \mathrm{c}) t=250 \mathrm{~ms}$. The arrows indicate the location of the singularities that progress along the transverse direction $y$. The size of the observation window is $32.5 \mathrm{~cm} \times 32.5 \mathrm{~cm}$.

from right to left. These images are taken from a high-speed movie recorded at a rate of $2.8 \mathrm{kHz}$. The vertical and the horizontal sizes of the visualization window are given by the size of the mirror. The wave appears as the juxtaposition of a white and a black line because of the focusing of light by the wave crest and its defocusing in the trough. The breaking of the wave is clearly visible on images b) and c) by the bending of the black line travelling ahead of the straight part of the wave with a slightly larger speed. The arrows of Figure 3-b) point the location of the two singularities propagating along the transversal direction $y$. Therefore the two lines separate in time, at least for the short durations of observation after the breaking started.

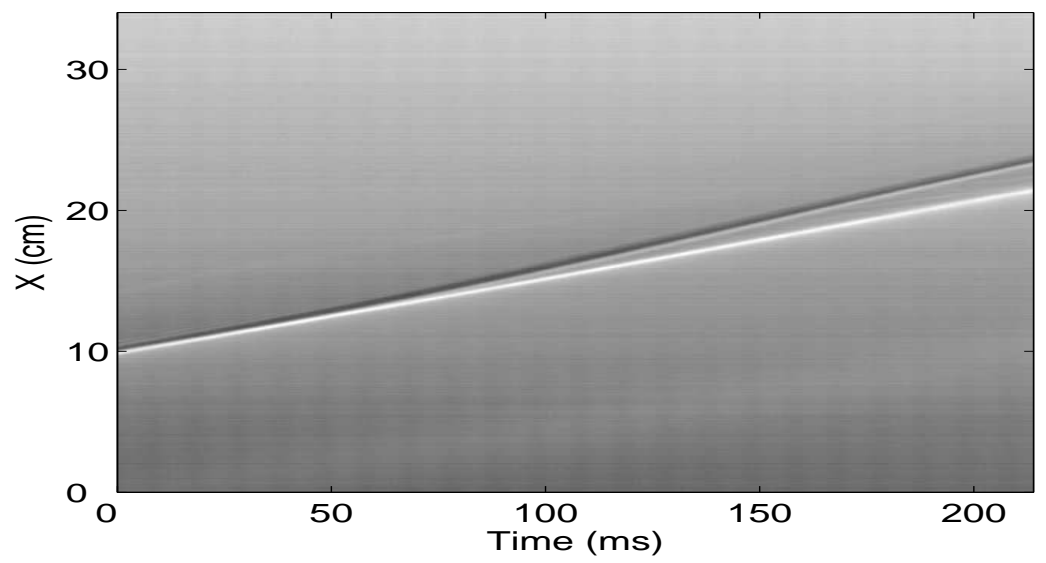

Fig. 4. Space-time diagram illustrating the progression of the wave along $x$ at a constant speed $v=0.41 \mathrm{~m} / \mathrm{s} \sim \sqrt{g h_{0}}$ (white line) and the appearance of the wave breaking (black bent line). Water depth $h_{0}$ is $17.4 \mathrm{~mm}$ and wave height is $8 \mathrm{~mm}$.

The longitudinal progression of the waves and of their breaking can also be particularly well illustrated by performing space-time diagrams. Taking a video 
horizontal line (corresponding to a given lateral position $y$ ) of each image and gathering them to reconstitute an image, illustrates the progression of the wave along the table. Figure 4 shows an example of such a space-time diagram where both the travelling wave and its breaking are visible. The white line represents the wave crest and rear face progression along the $x$ axis. As the height of the wave is constant on the distance it travels along the longitudinal coordinate $x$, it is not surprising to recover the constant gravitational wave speed $C_{B}=0.41 \mathrm{~m} / \mathrm{s} \sim \sqrt{g h_{0}}$ (the expected theoretical velocity should be $\left.C_{B}=0.46 \mathrm{~m} / \mathrm{s}\right)$. On the contrary, the black line which is the trace of the breaking during its progression along the table is slightly bent showing an acceleration of the breaking front ahead of the rest of the wave.

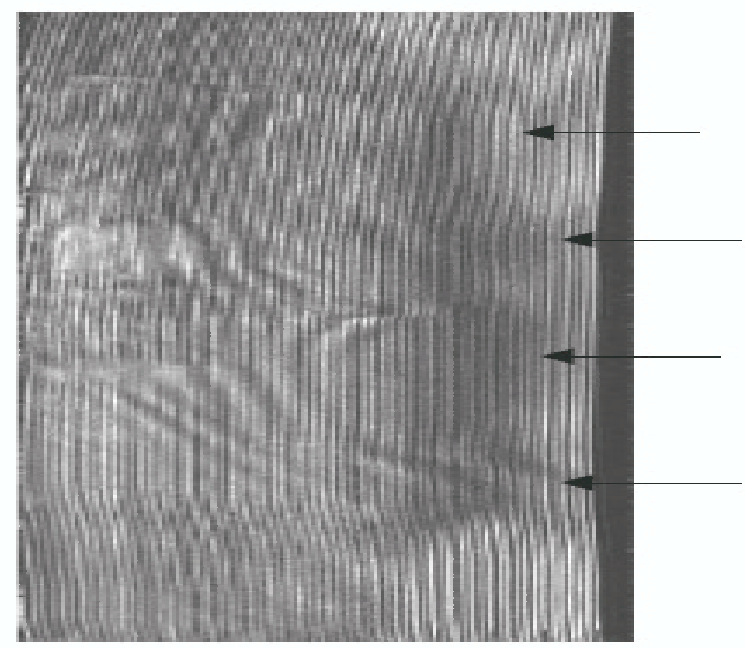

Fig. 5. Superimposition of images separated by $5 \mathrm{~ms}$ and showing the progression of the wave (enhanced by taking the horizontal gradient) that appears as vertical stripes. The wave breaking appears as a black shadow, mainly concentrated at the center of the figure. Time is proportional to the longitudinal coordinate and runs from right to left along the horizontal axis. Several sources of breaking are indicated by arrows before they merge. The size of this central observation window is $32.5 \mathrm{~cm}$ $\times 32.5 \mathrm{~cm}$.

As may be seen by close inspection of Figure 3, the breaking is initiated in the central region of the wave, far from the sidewall. We can then superimpose a complete series of images: as the wave travels at a constant speed, the longitudinal coordinate $x$ is then directly proportional to time. To increase the contrast of the result, the horizontal gradient of each images is taken. Figure 5 presents this superimposition where successive images are separated by a time delay of $5 \mathrm{~ms}$. It shows the progression of the wave from right to left at constant speed. The traces left by the wave appear as vertical stripes. The wave breaking appears as a black shadow, initiated at the center of the figure. As can be observed, several sources of breaking appear and progress laterally in time before merging to form a unique breaking front. To study the lateral progression of one breaking, and thus of the associated singularities, 


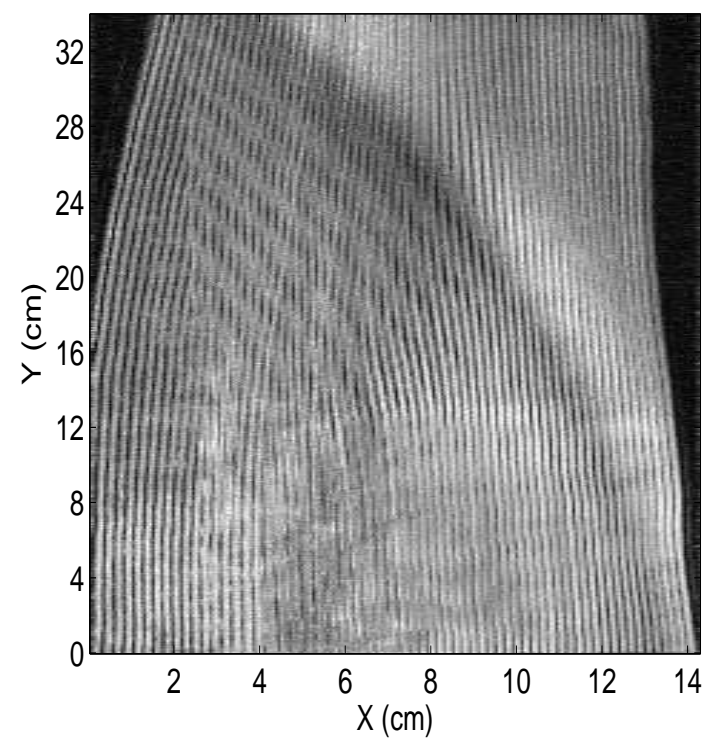

Fig. 6. Wave breaking trace as it progresses laterally towards the wall. Superimposition of images separated by $3 \mathrm{~ms}$. Horizontal axis is proportional to time that runs from right to left. The depth of water is $17.4 \mathrm{~mm}$ and the height of the wave is $8 \mathrm{~mm}$.

we focus on one side of the wave which is at the top of the images. For that, the mirror is simply moved from the center of the table to one side. Following the same image analysis as before, we record precisely the trace of the breaking waves that progress laterally. Figure 6 presents an example of the superimposition of images separated by $3 \mathrm{~ms}$. The position of the shadow is easily determined visually. It gives a curve which represents the lateral progression in time of the singularity associated with the breaking along the span-wise direction $y$ (see Figure 7-a). As can be observed on Figure 6 and Figure 7-a, the shape of this curve is by simple inspection very close to a square root and finally, after having determined the initial position $y_{0}$ of the breaking, a log-log representation of this curve is given in figure 7-b) and clearly confirms the square root nature of the lateral progression of the singularity as predicted in section 3 .

A systematic image analysis of our 50 cases of waves shows that this behavior, illustrated here on a single event, is truly generic: the breaking of the wave always progresses as the square root of time in the span-wise direction. We have also checked that the presence of a slight positive or negative slope (the table can be tilted by a few degrees in the longitudinal direction) has no significant effect on this behavior. However, even if all waves break systematically with the expected square root behavior, we have not found a clear evolution of the values of the coefficient $\gamma\left(y-y_{0}=\gamma \sqrt{t}\right)$ as functions of the wave height and/or the water depth. This coefficient depends on the initial conditions for the wave, depending on uncontrolled small perturbations of the wave crest. It 
a)

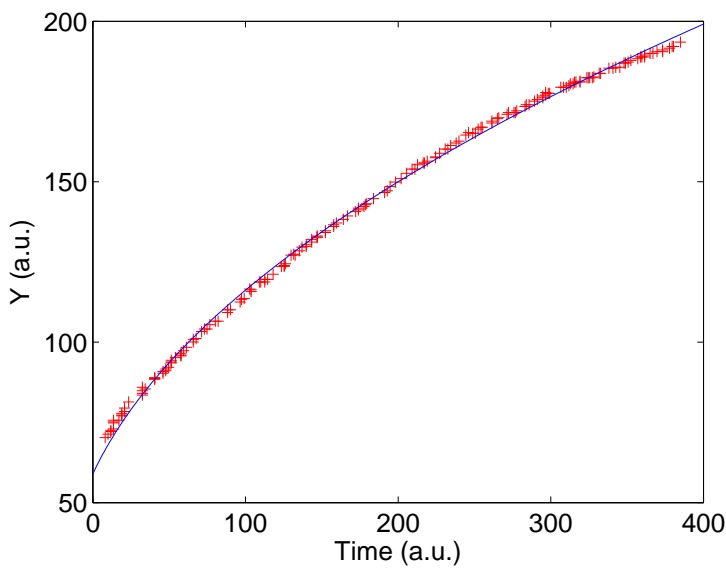

b)

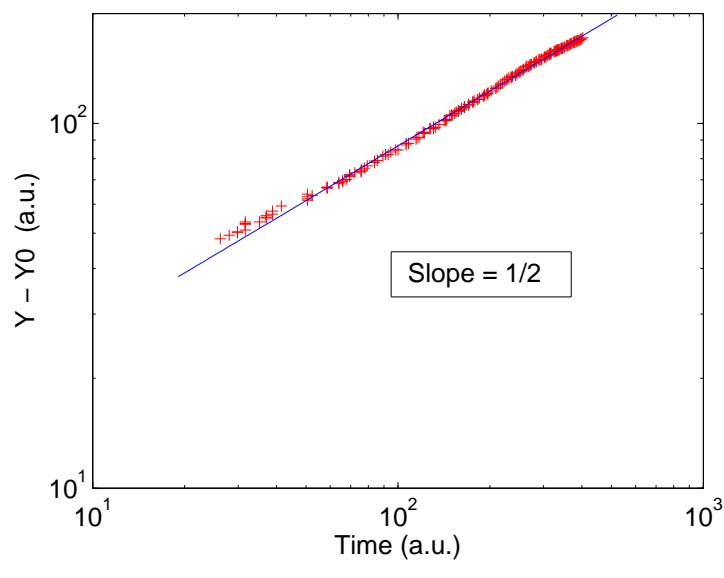

Fig. 7. a) Linear and b) logarithmic representation of the progression in time of the singularity along the lateral $y$ axis. The solid curve is a $2^{\text {nd }}$ order fit of the experimental data points.

could be also that the range of variations of water depth and wave height in our set-up was too limited to permit a significant change in the coefficient of the square root law.

\section{Summary and conclusion}

A generic analysis of wave-breaking predicts a widening of the crest with the square root of time after break-up begins. This is based on the general solution of the equations for a pressure-less inviscid fluid, and can be extended, at least near the break-up, to the fluid equations for long wave of relatively small amplitude and negligeable dispersion. Experiments carried in a shallow horizontal fluid layer show this square-root law for the breaking of solitary waves excited by a simple device. Therefore wave-breaking can be seen as belonging to the general class of phenomena described by nonlinear hyperbolic 
equations, realizing somehow the prediction made long ago by Riemann who viewed his solution of the equations of compressible gases more as a piece of mathematics than as something related to the reality of gases. As we know, he was wrong in this respect, because shock waves do exist in compressible gases, but his discovery has truly a wider range of application, in particular to waves in shallow water as we show here. This has been known for a long time of course, but as far as we are aware no prediction has been made for the widening of the crest of a wave. We believe that this provides an interesting domain of investigation because there the regularization (i.e. what happens after the singularity) is quite different from the one in the much studied cases of regularization by dispersion or by diffusion, the latter being relevant for shock waves in gases.

\section{References}

[1] V.I. Arnold, Yu.M. Baryshnikov and I.A. Bogayevsky, Supplement 2 on "Singularities and bifurcations of potential flows" in the book on "Nonlinear random waves and turbulence in nondispersive media" by S.N. Gurbatov et al., Manchester University Press, Manchester and New-York (1990).

[2] D.H. Peregrine, "Large-scale vorticity generation by breakers in shallow and deep water", Eur. J. Mech. b 18 (1999) pp. 403-408.

[3] V.A. Florin, Isvestia Ak.Nauk SSSR Otd.Tekhn.Nauk, 9 (1948) pp. 13891397.

[4] I. Newton, "The Mathematical principles of natural philosophy", trans. Andrew Motte (London, 1729) and "The principia: mathematical principles of natural philosophy Isaac Newton; a new translation" by I.B. Cohen and A. Whitman, University of California Press, Berkeley, Los Angeles \& London (1999).

[5] S.D. Poisson, "Mémoire sur la théorie du son", Journal de l'École Polytechnique, 14éme cahier, 7, 319-392 (1808). A short historical account of researches on the mathematical problem of generation of singularities in $1 \mathrm{D}$ nonlinear hyperbolic equations is to be found in Chapter III, p. 118-119 of R. Courant and K.O. Friedrichs "Supersonic flows and shock waves", Interscience Publishers, New York (1967).

[6] G. Stokes, "On the theory of oscillatory waves", Cambridge Phil.Trans., 8 (1847), 441-473.

[7] G. Whitham, Section 13.14 on breaking in "Linear and nonlinear waves" Wiley, New York (1974).

[8] Ya.B. Zel'dovich, Astrophysics, 6 (1970), pp 319-335. 